\title{
Solar origin of heliospheric magnetic field inversions: evidence for coronal loop opening within pseudostreamers
}

Article

Accepted Version

Owens, M. J., Crooker, N. U. and Lockwood, M. (2013) Solar origin of heliospheric magnetic field inversions: evidence for coronal loop opening within pseudostreamers. Journal of Geophysical Research: Space Physics, 118 (5). pp. 18681879. ISSN 2169-9402 doi: https://doi.org/10.1002/jgra.50259 Available at https://centaur.reading.ac.uk/32614/

It is advisable to refer to the publisher's version if you intend to cite from the work. See Guidance on citing.

Published version at: http://dx.doi.org/10.1002/jgra.50259

To link to this article DOI: http://dx.doi.org/10.1002/jgra.50259

Publisher: American Geophysical Union

All outputs in CentAUR are protected by Intellectual Property Rights law, including copyright law. Copyright and IPR is retained by the creators or other copyright holders. Terms and conditions for use of this material are defined in the End User Agreement.

\section{www.reading.ac.uk/centaur}


Central Archive at the University of Reading

Reading's research outputs online 
. Solar origin of heliospheric magnetic field inversions:

. Evidence for coronal loop opening within

3 pseudostreamers

\section{M.J. Owens}

4 Space Environment Physics Group, Department of Meteorology, University

${ }_{5}$ of Reading, Earley Gate, PO Box 243, Reading RG6 6BB, UK

N.U. Crooker

- Center for Space Physics, Boston University, Boston, MA 02215, USA

M. Lockwood

7 Space Environment Physics Group, Department of Meteorology, University

$s$ of Reading, Earley Gate, PO Box 243, Reading RG6 6BB, UK

M.J. Owens, Space Environment Physics Group, Department of Meteorology, University of Reading, Earley Gate, PO Box 243, Reading RG6 6BB, UK m.j.owens@reading.ac.uk 
9 Abstract. The orientation of the heliospheric magnetic field (HMF) in 10 near-Earth space is generally a good indicator of the polarity of HMF foot ${ }_{11}$ points at the photosphere. There are times, however, when the HMF folds ${ }_{12}$ back on itself (is inverted), as indicated by suprathermal electrons moving 


\section{Introduction}


as in Figure 1c, and in unipolar regions [e.g., Balogh et al., 1999], as in Figure 1d These pairs of field changes bound inversions that are usually of short duration, on the order of an hour or two. In contrast, inversions bounded on at least one side by a change in the strahl direction with no change in the magnetic field direction are less common but can be of long duration, on the order of a day or more [Crooker et al., 2004b]. Moreover, they can only be understood in terms of a three-dimensional structure. In cases involving the HCS, as in Figure 1e, where the dashed field lines lie out of the plane of the Figure, the inversion results in a mismatch between the magnetic and electron signatures of the sector boundary [Crooker et al., 2004b].

While some of the smaller inversions may be the product of large-scale turbulent processes, the larger inversions appear to be robust signatures of near-Sun magnetic interchange reconnection, as sketched in Figures 1c-e, where a green X marks a reconnection site. The legs of large loops expanding into the heliosphere reconnect with adjacent open field lines. Crooker et al. [2004b] suggest that the expanding loops are at the quiet end of a spectrum of large-scale transient outflows, with coronal mass ejections (CMEs) at the active end. This interpretation is supported by the observation of coronal inflows and collapsing loops at locations where the HCS is inclined to the solar rotation direction [Sheeley and Wang, 2001], taken to be signatures of magnetic reconnection. The association of inverted HMF with the HCS suggests the solar origin of the expanding loops can be bipolar helmet streamers which surround the coronal source-surface neutral line and separate magnetic flux from coronal holes of opposite magnetic polarity, e.g., the 


\section{Detection of HMF inversions}

February 7, 2013, 2:23pm 
netic polarity predicted by the PFSS model and that observed near-Earth. Green crosses show the coronal source-surface locations of observed HMF inversions at the heliographic latitude of Earth.

The two intervals of inverted HMF at Carrington longitude of . The remaining HMF inversions are also associated with a change in magnetic connectivity, with the photospheric foot points along Earth orbit shifting between different coronal holes, but without an associated change in foot point polarity, indicative of pseudostreamers. These HMF inversions are thus associated with pseudostreamers rather than bipolar streamers.

define a parameter $d S$, the distance between photospheric foot points of neighbouring points on the source surface. In practice, the magnitude of $d S$ will depend on the spatial resolution at which field lines are traced, making units somewhat arbitrary. In this study, we calculate $d S$ by moving along the ecliptic plane in $1^{\circ}$ steps. When adjacent points on the source surface map to the same coronal hole, $d S$ will be small, for example as seen between $0^{\circ}$ and $60^{\circ}$ Carrington longitude for CR1990. When neighbouring source-surface points map to different coronal holes, however, such as the HCS crossing at $310^{\circ}$ Carrington longitude, $d S$ will be very large. The middle panel of Figure 4 shows $\log _{e}(d S)$ as a function of Carrington longitude along the ecliptic plane. Vertical yellow lines mark HCS crossings, where $\log _{e}(d S)$ spikes correspond to bipolar streamers. The dashed horizontal line at $\log _{e}(d S)=3$ marks the threshold selected to define a streamer. It is the value which $\log _{e}(d S)$ reaches or exceeds at all HCS crossings in the 1998 to 2011 period and corresponds to source surface points with a $1^{\circ}$ separation having a photospheric footpoint separation of $\geq 5^{\circ}$. It thus selects all bipolar streamers and appears to select most sig- 
nificant pseudostreamers while suppressing smaller structures. Blue vertical lines mark $\log _{e}(d S)$ spikes without polarity reversals, our definition of a pseudostreamer. The 17 1-hour intervals of inverted HMF not associated with the HCS in CR1990 all map close to the longitudes of pseudostreamers.

The bottom panel of Figure 4 is a contour plot of $d S$ at all latitudes. It demonstrates in another way the finding reported by [Crooker et al., 2012] that pseudostreamer belts but connect to the bipolar streamer belt to form a network of slow solar wind sources that expands to cover the source surface during solar maximum. As is the case for bipolar streamers, HMF inversions are not associated with all pseudostreamers; however, Figure 4 demonstrates that streamer-associated inverted HMF is likely to be common at all latitudes near solar maximum.

\subsection{Statistical analysis}

In order to systematically analyse the entire 1998-2011 interval, and define strict thresholds for association between inverted HMF and streamers. We begin by including only Carrington rotations in which the PFSS model provides a reasonable representation of the observed magnetic structure of the corona and solar wind. By assigning $+1(-1)$ to outward (inward) Parker spiral polarity, and ignoring undetermined, counterstreaming and inverted intervals, we compute the mean-square error (MSE) between the PFSS and observed sector structure mapped to the source surface. Thus MSE is a combination of errors in the PFSS solution and errors in the simple ballistic mapping of near-Earth solar wind to the coronal source surface. 
of ecliptic longitudes are covered by pseudostreamers (bipolar streamers). Note that the association scheme allows a single inverted HMF interval to map to both a bipolar and pseudostreamer if they are located close in longitude. Table 2 summarises these results.

In general, there are insufficient inverted HMF events to detect significant differences in the of solar wind properties of bipolar- and pseudostreamer-associated inversions. Probability distributions of density, however (not shown), suggest that HMF inversions from bipolar streamers contain denser solar wind than inverted HMF from pseudostreamers, consistent with general properties of pseudostreamer-associated solar wind [Wang et al., $2012]$

\section{Conclusions and Discussion}

The polarity of the photospheric foot point of heliospheric magnetic flux (HMF) can be independently estimated from both the local HMF orientation, as measured using in situ magnetometer observations, and the direction of the suprathermal electron beam, or "strahl." For the bulk of the solar wind, these two methods show agreement. There are intervals, however, in which the strahl is directed towards the Sun, implying that the magnetic field line is inverted, or folded back on itself. This is an expected signature of near-Sun magnetic reconnection by which the Sun can open previously closed heliospheric loops [Owens et al., 2011; Owens and Lockwood, 2012]. Using an automated data analysis method, we find inverted flux in approximately 5.5\% of the solar wind data between 1998 and 2011, though this is likely an underestimate due to strict selection criteria. We do not find a strong solar cycle variation in the occurrence rate of inverted HMF, but this finding is confined to the ecliptic plane. Inverted HMF is associated with dense, slow, cool solar wind, with lower than average magnetic field intensity. In order to determine 
infer the global structure of the coronal magnetic field and a new automated detection method for bipolar and pseudostreamers. Of the 2263 1-hour inverted HMF intervals identified in the solar wind and mapped back to the coronal source surface, 1310 (58\%) are associated with streamers. Given that the probability of a solar wind interval being associated with a streamer by chance is $52 \%$, the association between inverted HMF and streamers is significant at the $99.9 \%$ level. Of the 1310 streamer-associated inverted HMF intervals, 949 (504) map to pseudostreamers (bipolar streamers). This ratio is in reasonable agreement with the occurrence rates of pseudostreamers and bipolar streamers in the ecliptic plane, $39 \%$ and $20 \%$, respectively,

If we assume that inverted HMF is primarily a signature of reconnection in the corona [e.g., Titov et al., 2011], our results suggest that the rate of reconnection is similar within bipolar and pseudostreamers. This seems reasonable in view of their magnetic structure. For the bipolar streamer case, a three-dimensional magnetic configuration for interchange reconnection that can create the inversion is illustrated in 1e and has already been discussed in section 1. For the pseudostreamer case, an appropriate magnetic configuration can be drawn in just two dimensions, as illustrated in Figure 6. Closed loops within one of the two arcades that form pseudostreamers are shown to rise as a result of photospheric flux emergence, but could equally be the result of loop foot point shearing, etc. In the top panel, the rising loop undergoes interchange reconnection before it reaches the solar wind acceleration height and therefore doesn't result in the generation of inverted HMF. This configuration is common from the solar perspective [e.g., Wang et al., 2007; Crooker et al., 2012]. In contrast, from the heliospheric perspective, the rising loops are dragged 
Smith, Solar wind streamer belt structure, J. Geophys. Res., 101, 24,331-24,342, doi: 10.1029/96JA02412, 1996.

Crooker, N. U., J. T. Gosling, and S. W. Kahler, Magnetic clouds at sector boundaries, J. Geophys. Res., 103, 301, 1998.

Crooker, N. U., C.-L. Huang, S. M. Lamassa, D. E. Larson, S. W. Kahler, and H. E. Spence, Heliospheric plasma sheets, J. Geophys. Res., 109, A03107, doi: 10.1029/2003JA010170, 2004a.

Crooker, N. U., S. W. Kahler, D. E. Larson, and R. P. Lin, Large-scale magnetic field inversions at sector boundaries, J. Geophys. Res., 109, doi:10.1029/2003JA010278, 2004b.

Crooker, N. U., S. K. Antiochos, X. Zhao, and M. Neugebauer, Global network of slow solar wind, J. Geophys. Res., 117, A04104, doi:10.1029/2011JA017236, 2012.

Erdős, G., and A. Balogh, Magnetic Flux Density Measured in Fast and Slow Solar Wind Streams, Astrophys. J., 753, 130, doi:10.1088/0004-637X/753/2/130, 2012.

Eselevich, V. G., On the structure of coronal streamer belts, J. Geophys. Res., 103, 2021, doi:10.1029/97JA02365, 1998.

Eselevich, V. G., V. G. Fainshtein, and G. V. Rudenko, Study of the structure of streamer belts and chains in the solar corona, 188, 277-297, 1999.

Fainshtein, V. G., An Investigation of Solar Factors Governing Coronal Mass Ejection Characteristics, 174, 413-435, 1997.

Feldman, W. C., J. R. Asbridge, S. J. Bame, M. D. Montgomery, and S. P. Gary, Solar wind electrons, J. Geophys. Res., 80, 4181-4196, 1975.

Fisk, L. A., Acceleration of the solar wind as a result of the reconnection of open magnetic flux with coronal loops, J. Geophys. Res., 108, 1157, doi:10.1029/2002JA009284, 2003. 
Liu, Y., and K. Hayashi, The 2003 October-November Fast Halo Coronal Mass Ejections and the Large-Scale Magnetic Field Structures, Astrophys. J., 640, 1135-1141, doi: $10.1086 / 500290,2006$.

Lockwood, M., M. Owens, and A. P. Rouillard, Excess open solar magnetic flux from satellite data: 2. A survey of kinematic effects, J. Geophys. Res., 114, A11104, doi: 10.1029/2009JA014450, 2009.

McComas, D. J., J. T. Gosling, D. Winterhalter, and E. J. Smith, Interplanetary magnetic field draping about fast coronal mass ejecta in the outer heliosphere, J. Geophys. Res., 93, 2519-2526, doi:10.1029/JA093iA04p02519, 1988.

McComas, D. J., S. J. Bame, B. S. J., W. C. Feldman, J. L. Phillips, P. Riley, and J. W. Griffee, Solar wind electron proton alpha monitor (SWEPAM) for the Advanced Composition Explorer, Space Sci. Rev., 86, 563, 1998.

Nash, A. G., N. R. Sheeley, Jr., and Y.-M. Wang, Mechanisms for the rigid rotation of coronal holes, Sol. Phys., 117, 359-389, 1988.

Owens, M. J., and P. J. Cargill, Non-radial solar wind flows induced by the motion of interplanetary coronal mass ejections, Ann. Geophys., 22, 4397-4395, 2004.

Owens, M. J., and N. U. Crooker, Coronal mass ejections and magnetic flux buildup in the heliosphere, J. Geophys. Res., 111, A10104, doi:10.1029/2006JA011641, 2006.

Owens, M. J., and N. U. Crooker, Reconciling the electron counterstreaming and dropout occurrence rates with the heliospheric flux budget, J. Geophys. Res., 112, A06106, doi: 10.1029/2006JA012159, 2007.

Owens, M. J., and M. Lockwood, Cyclic loss of open solar flux since 1868: The link to heliospheric current sheet tilt and implications for the Maunder Minimum, J. Geo- 
phys. Res., 117, A04102, doi:10.1029/2011JA017193, 2012.

Owens, M. J., N. A. Schwadron, N. U. Crooker, W. J. Hughes, and H. E. Spence, Role of coronal mass ejections in the heliospheric Hale cycle, Geophys. Res. Lett., 34, L06104, doi:10.1029/2006GL028795, 2007.

Owens, M. J., C. N. Arge, N. U. Crooker, N. A. Schwadron, and T. S. Horbury, Estimating total heliospheric magnetic flux from single-point in situ measurements, J. Geophys. Res., 113, A12103, doi:10.1029/2008JA013677, 2008a.

Owens, M. J., N. U. Crooker, N. A. Schwadron, T. S. Horbury, S. Yashiro, H. Xie, O. C. St Cyr, and N. Gopalswamy, Conservation of open solar magnetic flux and the floor in the heliospheric magnetic field, Geophys. Res. Lett., L20108, doi:10.1029/2008GL035813, 2008b.

Owens, M. J., N. U. Crooker, and M. Lockwood, How is open solar magnetic flux lost over the solar cycle?, J. Geophys. Res., 116, A04111, doi:10.1029/2010JA016039, 2011.

Phan, T. D., et al., A magnetic reconnection X-line extending more than 390 Earth radii in the solar wind, Nature, 439, 175-178, doi:10.1038/nature04393, 2006.

Richardson, I. G., and H. V. Cane, Near-earth solar wind flows and related geomagnetic activity during more than four solar cycles (1963-2011), J. Geophys. Res., 2(26), A02, doi:10.1051/swsc/2012003, 2012.

Riley, P., and J. G. Luhmann, Interplanetary Signatures of Unipolar Streamers and the Origin of the Slow Solar Wind, Sol. Phys., 277, 355-373, doi:10.1007/s11207-011-9909-0, 2012.

Rosenbauer, H., et al., A survey on initial results of the HELIOS plasma experiment, Journal of Geophysics Zeitschrift Geophysik, 42, 561-580, 1977. 

magnetic fields, Sol. Phys., 9, 442-455, 1969.

Sheeley, N. R., Jr., and Y.-M. Wang, Coronal Inflows and Sector Magnetism, Astrophys. J. Lett., 562, L107-L110, doi:10.1086/338104, 2001.

Smith, C. W., J. L'Heureux, N. F. Ness, M. H. Acuna, L. F. Burlaga, and J. Scheifele, The ACE magnetic fields experiment, Space Sci. Rev., 86, 613, 1998.

Steinberg, J. T., J. T. Gosling, R. M. Skoug, and R. C. Wiens, Suprathermal electrons in high-speed streams from coronal holes: Counterstreaming on open field lines at 1 AU, J. Geophys. Res., 110, A06103, doi:10.1029/2005JA011027, 2005.

Titov, V. S., Z. Mikić, J. A. Linker, R. Lionello, and S. K. Antiochos, Magnetic Topology of Coronal Hole Linkages, Astrophys. J., 731, 111, doi:10.1088/0004-637X/731/2/111, 2011.

Wang, Y., and N. R. Sheeley, Jr., Footpoint Switching and the Evolution of Coronal Holes, Astrophys. J., 612, 1196-1205, doi:10.1086/422711, 2004.

Wang, Y.-M., and N. R. Sheeley, Jr., Solar Implications of ULYSSES Interplanetary Field Measurements, Astrophys. J. Lett., 447, L143-L146, doi:10.1086/309578, 1995.

Wang, Y.-M., N. R. Sheeley, Jr., and N. B. Rich, Coronal Pseudostreamers, Astrophys. J., 658, 1340-1348, doi:10.1086/511416, 2007.

Wang, Y.-M., R. Grappin, E. Robbrecht, and N. R. Sheeley, Jr., On the Nature of the Solar Wind from Coronal Pseudostreamers, Astrophys. J., 749, 182, doi:10.1088/0004$637 \mathrm{X} / 749 / 2 / 182,2012$.

Wimmer-Schweingruber, R. F., et al., Understanding interplanetary coronal mass ejection signatures, Space Sci. Rev., 123, 177-216, doi:10.1007/s11214-006-9017-x, 2006. 
${ }_{404}$ Zhao, X. P., and D. F. Webb, Source regions and storm effectiveness of frontside full halo ${ }^{405}$ coronal mass ejections, J. Geophys. Res., 108, 1234, doi:10.1029/2002JA009606, 2003. 


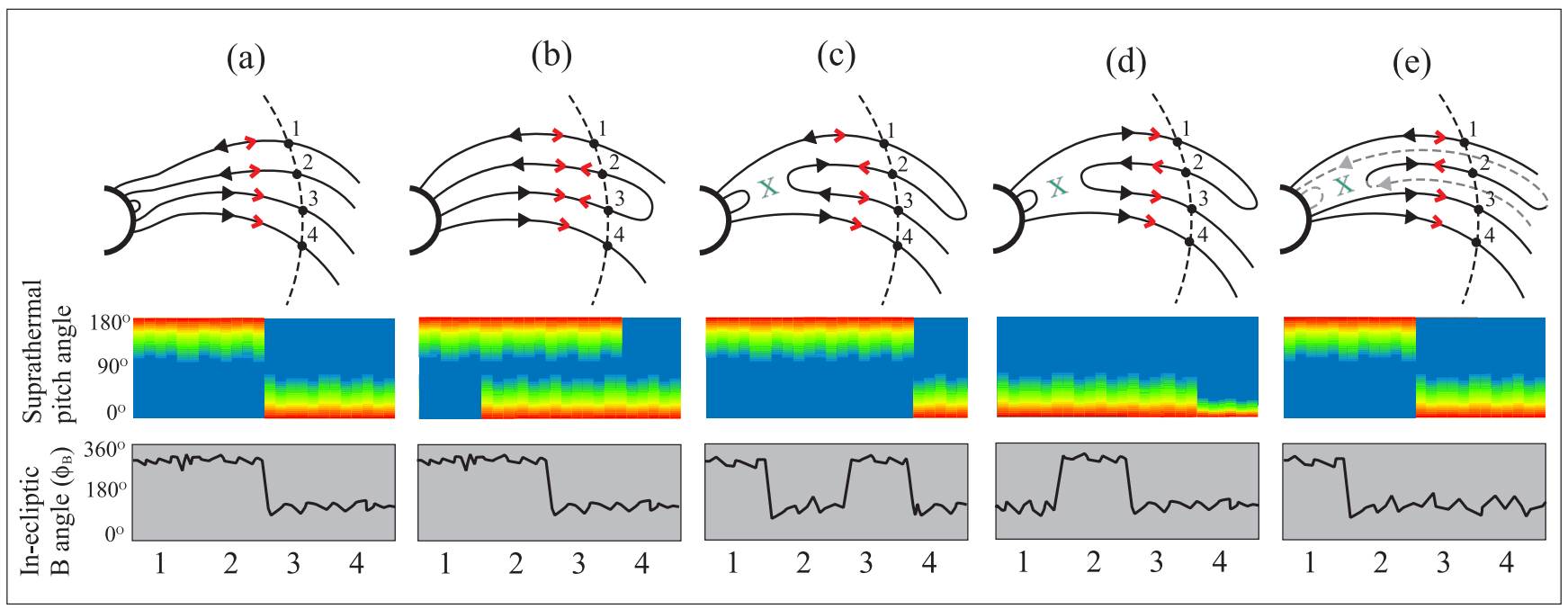

Figure 1. Sketches of possible HMF configurations and the resulting magnetic field and suprathermal electron signatures in near-Earth space. Red (black) arrows show the supratherthermal electron strahl (magnetic field polarity), while green crosses show the position of magnetic reconnection. (a) A typical sector boundary/HCS crossing. (b) A sector boundary accompanied by closed HMF loops, likely part of an ICME. (c) A sector boundary/HCS crossing containing an inverted HMF interval at time 2. (d) An inverted HMF interval at time 2 embedded within a unipolar region. (e) A sector boundary with mismatched electron and magnetic signatures. The dashed lines show portions of the inverted HMF structure which are out of the ecliptic plane and not encountered by the observing spacecraft [after Crooker et al., 2004b]. 


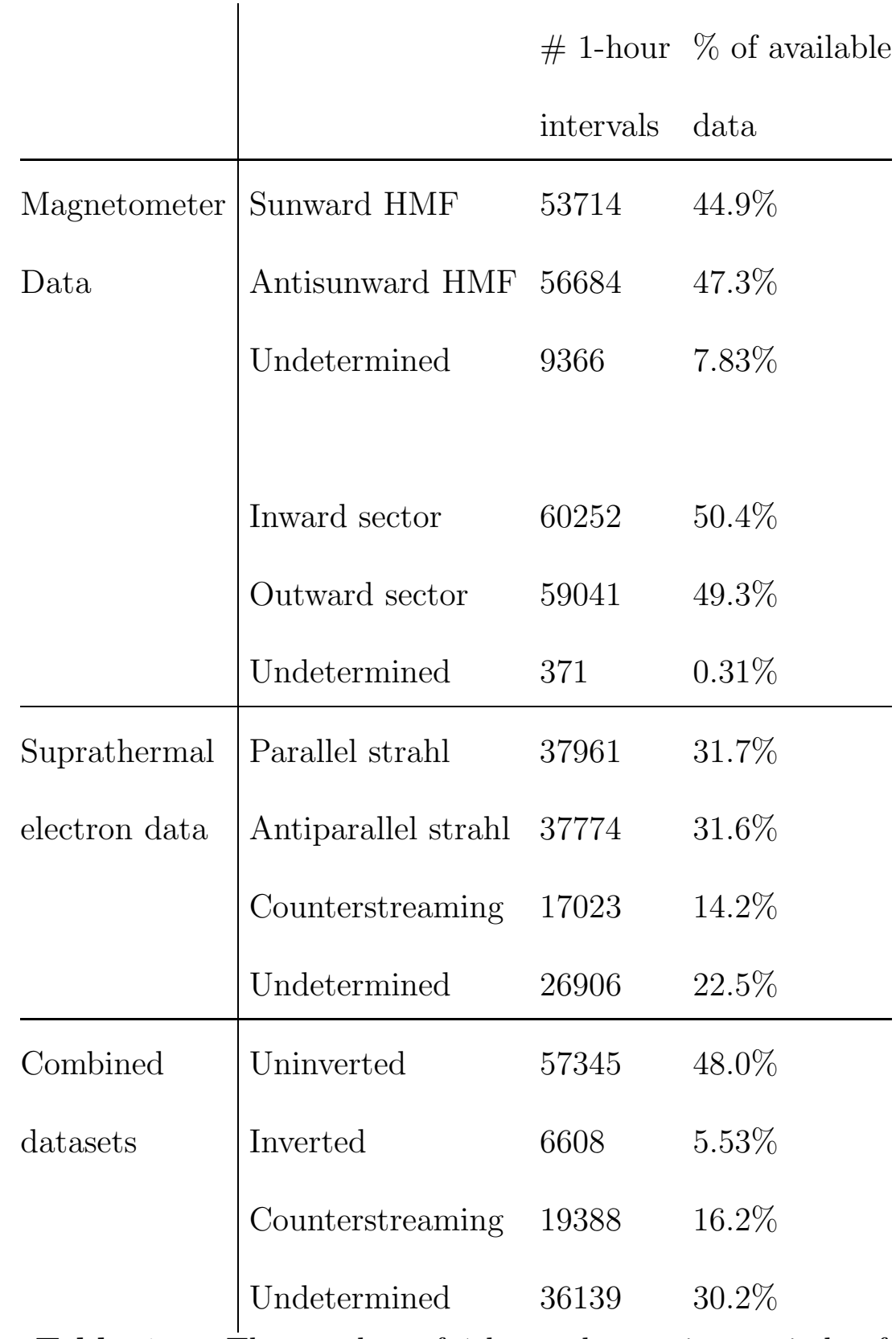

Table 1. The number of 1-hour observation periods of different HMF populations obtained using the magnetic field and suprathermal electron selection criteria. 


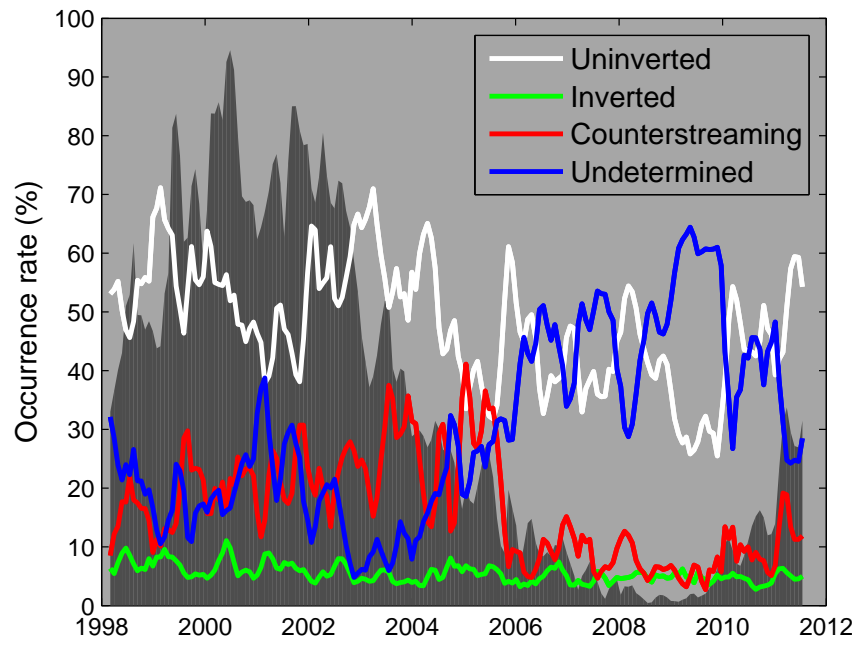

Figure 2. Three-Carrington rotation averages of the occurrence rates of various HMF topologies as a function of time. Sunspot number, scaled to fit the axis, is shown as the dark shaded region. Although some changes in the various HMF populations are likely to be due to changes in the electron detector, what this figure makes clear is that inverted flux is detected throughout the solar cycle. 

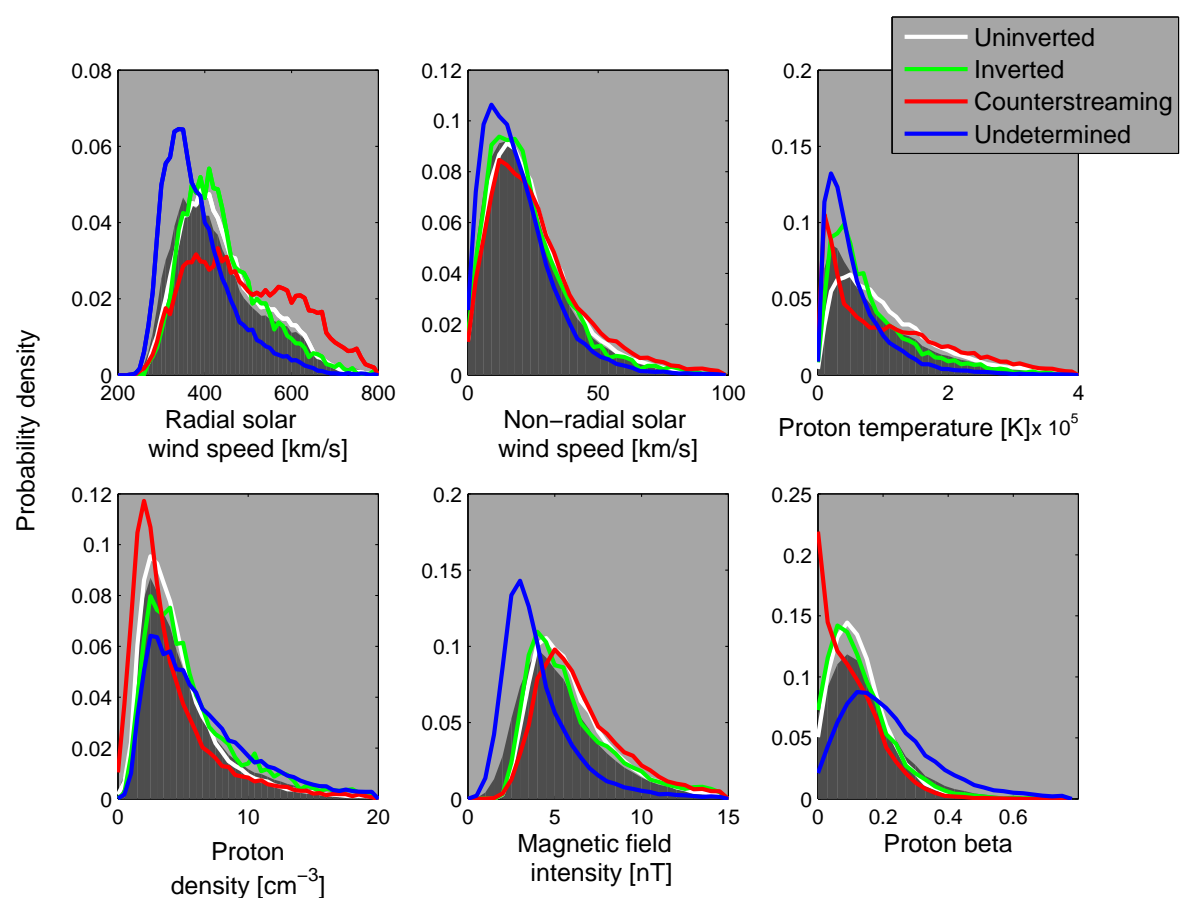

Figure 3. Probability distribution functions for various near-Earth solar wind populations. The grey shaded region shows all solar wind in the interval 1998-2011. Coloured lines show subsets of these data: White, green, red and blue lines show uninverted, inverted, counterstreaming and undetermined HMF intervals, respectively. 


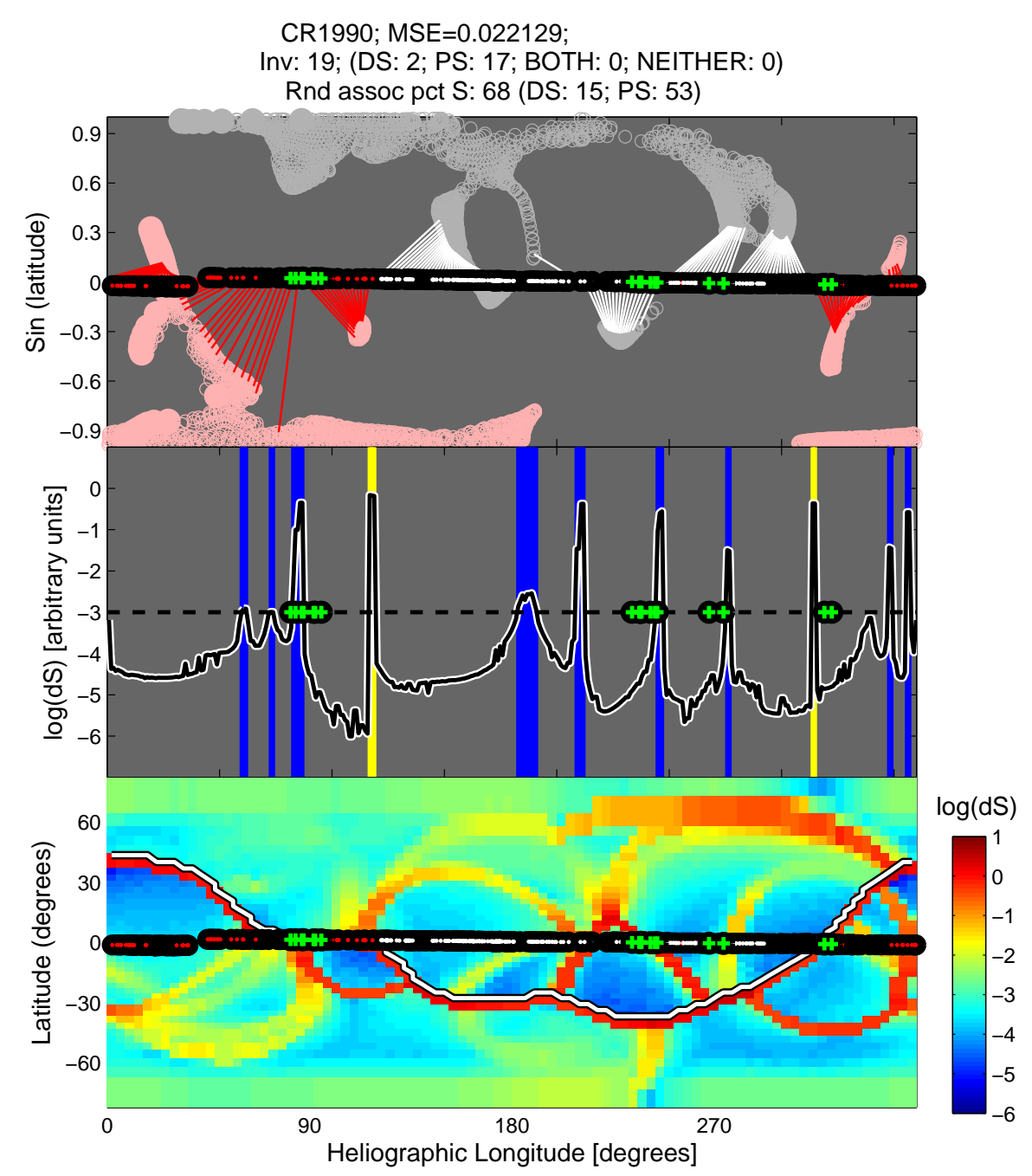

Figure 4. Top: A latitude-longitude map of the PFSS solution for Carrington rotation 1990. Pink/dark grey regions are the PFSS inward/outward coronal holes, with red/white lines showing the connection between the Earth's orbit across the source surface and photosphere. Overlaid on the black strip are red/white dots showing the observed outward/inward sectors mapped to the source surface. Green crosses are inverted flux intervals. Middle: dS, photospheric foot point separation for adjacent points on the source surface, along the ecliptic plane (shown on a $\log _{e}$ scale). This parameter serves as a means of identifying coronal streamers: Bipolar (pseudo) streamers are shown as vertical yellow (blue) lines. Bottom: contour plot of dS over all latitudes of the source surface. The HCS is the white curve. 
CR2011; MSE $=0.04756$;

Inv: 27; (DS: 6; PS: 15; BOTH: 0; NEITHER: 6)

Rnd assoc pct S: 56 (DS: 16; PS: 41)

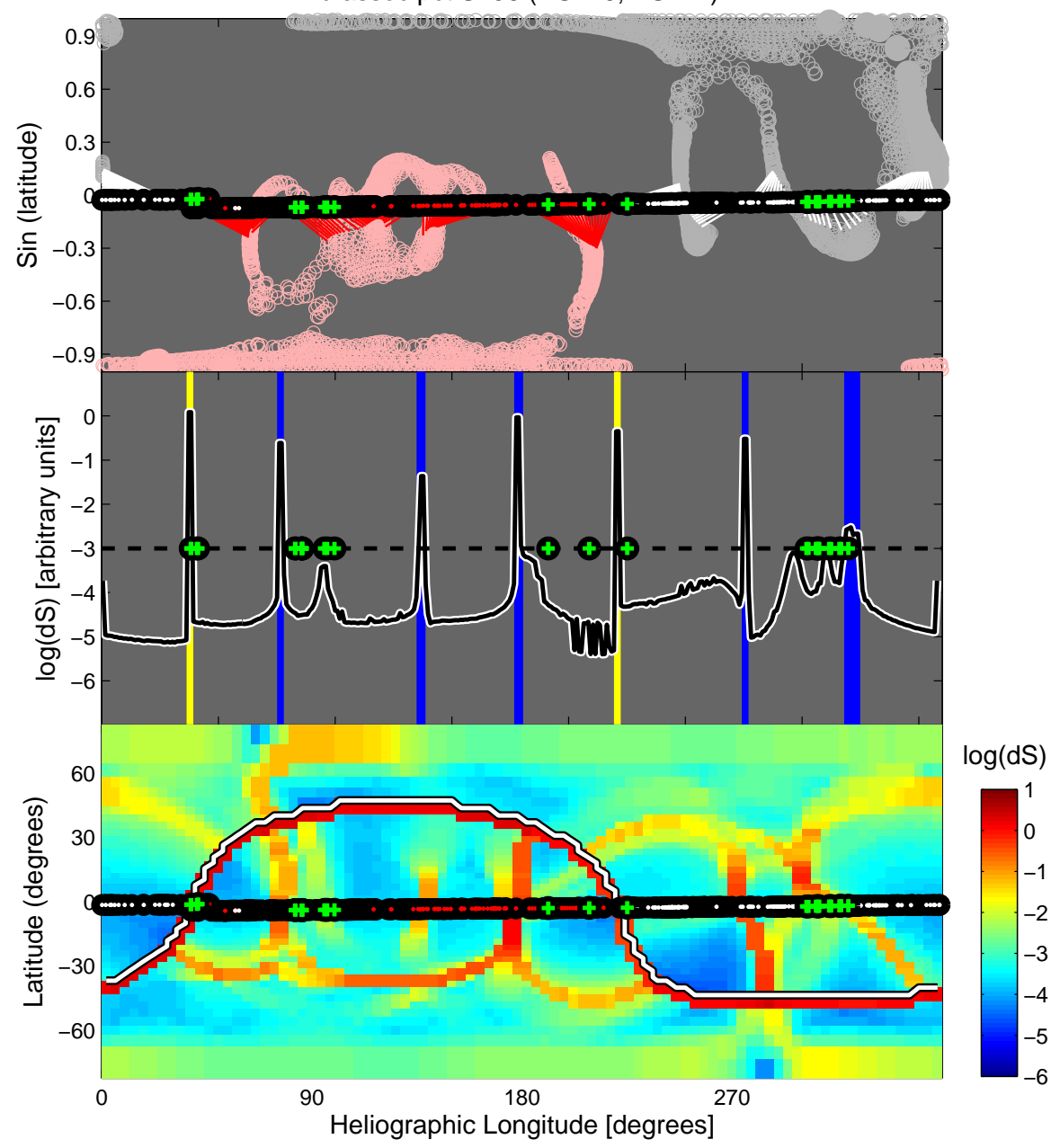

Figure 5. Parameters for Carrington rotation 2011, in the same format as Figure 4. 


\begin{tabular}{l|cccccc}
\hline & Total & Any & Pseudo & Bipolar & Both PS & No streamer \\
& & streamer & $(\mathrm{PS})$ & $(\mathrm{DS})$ & and DS & association \\
\hline Inverted HMF & 2263 & 1310 & 949 & 504 & 143 & 953 \\
$(\%$ of total $)$ & - & $(57.9 \%)$ & $(41.9 \%)$ & $(22.3 \%)$ & $(6.3 \%)$ & $(42.1 \%)$ \\
\hline Random & - & $52.4 \%$ & $39.0 \%$ & $20.5 \%$ & $5.1 \%$ & $47.6 \%$ \\
interval & & & & & & \\
\hline
\end{tabular}

Table 2. Solar origins of the inverted HMF intervals. Also shown is the probability that a random solar wind interval would be associated with the given type of streamer, i.e., the percentage of ecliptic longitudes which are associated with different coronal structures. 
(a) Pseudostreamer loop does not extend to solar wind formation height

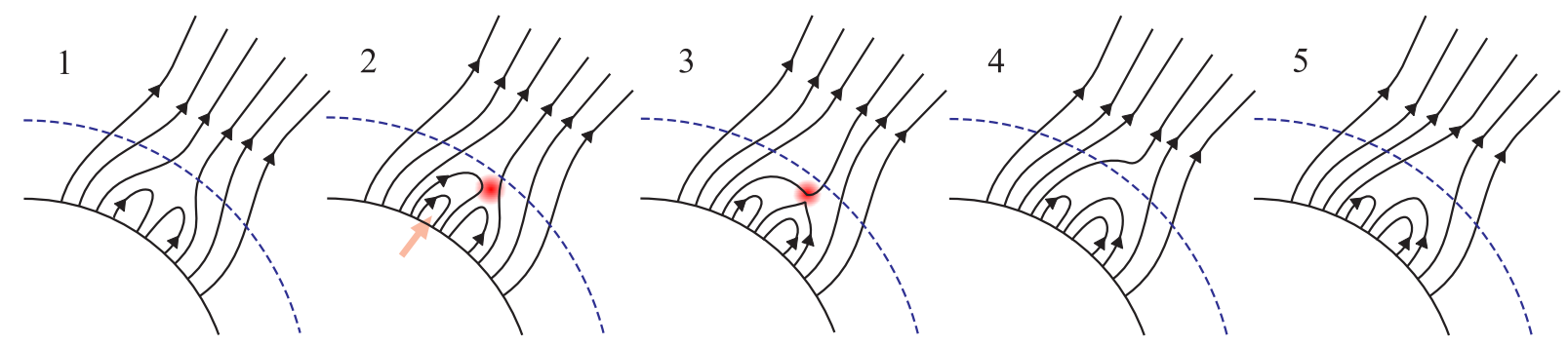

(b) Pseudostreamer loop extends to solar wind formation height

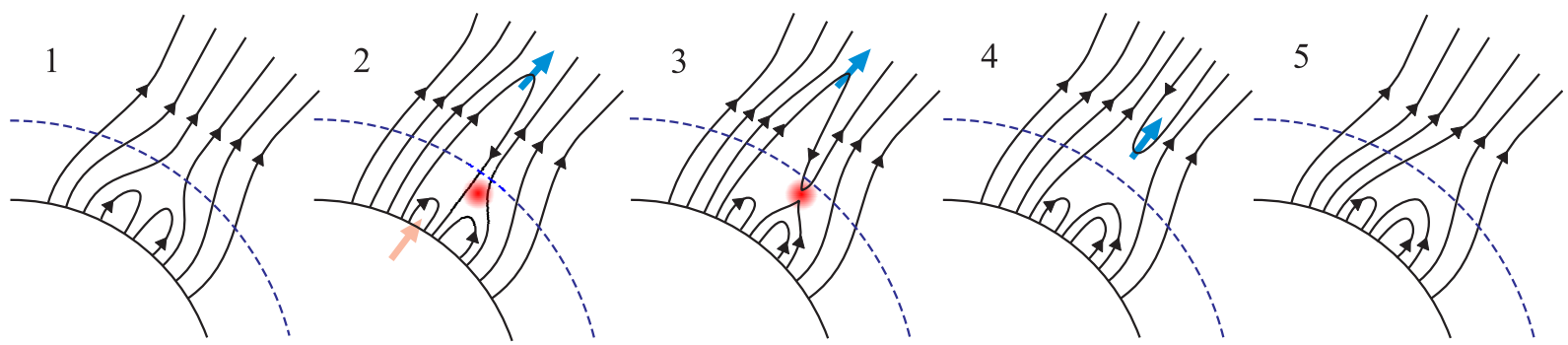

Figure 6. A sketch of of interchange reconnection within a pseudostreamer. In the top panel, a closed loop rises due to photospheric flux emergence (red arrow), but does not the reach the solar wind acceleration height (blue dashed line) before it undergoes reconnection with an open magnetic field line. This creates an Alfven wave on the open magnetic field line which propagates out into the heliosphere, but does not create inverted HMF. The bottom panels show a loop which is dragged out by the solar wind (blue arrow) before interchange reconnection occurs. It does result in the creation of inverted HMF. 